\title{
Biomarkers for predicting COPD exacerbations
}

This Danish prospective cohort study compared frequency of exacerbations in stable chronic obstructive pulmonary disease (COPD) patients with chronically raised inflammatory biomarkers to those with normal inflammatory biomarkers over a 5 -year follow-up. It measured baseline levels of C-reactive protein (CRP), leucocytes and fibrinogen levels.

An exacerbation was defined as requiring a short course of treatment with oral corticosteroids and/or antibiotics, or requiring hospital treatment due to COPD. Frequent exacerbations were defined as having two or more per year and each being more than 4 weeks apart.

This study found that simultaneously elevated levels of CRP, fibrinogen and leucocytes positively correlated with an increased risk of frequent exacerbations in stable COPD, which was statistically significant. The relative risk remained consistent when multivariable factors, such as previous exacerbations, symptom burden, disease severity and Body Mass Index were included, indicating that inflammatory biomarkers give additional information when riskstratifying patients.

Although this was a large prospective study with $100 \%$ follow-up rate, a large percentage of patients suffered with mild COPD, and were not on regular inhaled therapy. Therefore, the numbers with frequent exacerbations were small, decreasing the statistical power of the results.

Currently, the main predictors for frequent exacerbations are previous exacerbation and disease severity. This study suggests that measuring inflammatory biomarkers in stable disease enables clinicians to further risk-stratify their patients and potentially provide high-risk patients with preventative medication. However, further investigation is needed to determine the clinical value of these biomarkers for risk stratification.

- Thomsen M, Ingebrigtsen TS, Marott JL, et al. Inflammatory biomarkers and exacerbations in chronic obstructive pulmonary disease. JAMA 2013:309:2353-61.

Ruth Pais

Correspondence to Dr Ruth Pais; ruth.a.pais@gmail.com

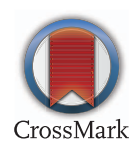

To cite Pais R. Thorax 2014;69:767.

Published Online First 7 September 2013

Thorax 2014;69:767. doi:10.1136/thoraxjnl-2013-204442 